\title{
Fatores facilitadores e dificultadores para a prática de notificação de eventos adversos por enfermeiros
}

\author{
Facilitating and hindering factors for the practice of reporting adverse events by nurses \\ Factores facilitadores y obstaculizadores para la práctica de reportar eventos adversos \\ por parte de enfermeras
}

Júnia Aparecida Pereira ${ }^{1}$, Marilane de Oliveira Fani Amaro ${ }^{1 *}$, Érica Toledo de Mendonça1, Camila Santana Domingos ${ }^{1}$, Andréia Guerra Siman ${ }^{1}$, Camilo Amaro de Carvalho ${ }^{1}$.

\section{RESUMO}

Objetivo: Identificar os fatores facilitadores e dificultadores envolvidos no processo de notificação de eventos adversos em saúde realizadas pelos enfermeiros que atuam em um hospital da Zona da Mata Mineira. Métodos: Estudo de caso qualitativo realizado por meio de entrevista com roteiro semiestruturado com a participação de 25 enfermeiros. Os dados foram submetidos a análise de conteúdo. Resultados: A partir dos depoimentos, emergiram duas categorias temáticas: Aspectos facilitadores para a realização da notificação de eventos adversos à saúde e "Notificação de eventos adversos: desafios vivenciados pela equipe de enfermagem". A primeira categoria evidenciou a comunicação eficaz, o trabalho em equipe e o aprendizado a partir da notificação como aspectos que facilitam a realização das notificações. Por sua vez, a segunda categoria apontou a cultura punitiva, a falta de feedback e a sobrecarga de trabalho como aspectos dificultadores. Conclusão: Os profissionais de saúde em sua prática cotidiana, vivenciam a ocorrência de eventos adversos e reconhecem que o processo de notificação é um aspecto fundamental para a promoção da segurança do paciente. Conhecer e determinar a real magnitude dos eventos adversos, é questão primordial para uma gestão de qualidade nas instituições promotoras do cuidado.

Palavras-chave: Segurança do paciente, Evento adverso, Enfermagem, Notificação.

\begin{abstract}
Objective: To identify the facilitating and hindering factors involved in the process of notification of adverse health events carried out by nurses who work in a hospital in Zona da Mata Mineira. Methods: Qualitative case study conducted through an interview with a semi-structured script with the participation of 25 nurses. The data were submitted to content analysis. Results: From the testimonies, two thematic categories emerged: Facilitating aspects for carrying out the notification of adverse health events and "Notification of adverse events: challenges experienced by the nursing team". The first category highlighted effective communication, teamwork and learning from notification as aspects that facilitate the realization of notifications. In turn, the second category pointed out the punitive culture, lack of feedback and work overload as hindering aspects. Conclusion: Health professionals in their daily practice, experience the occurrence of adverse events and recognize that the notification process is a fundamental aspect for the promotion of patient safety. Knowing and determining the real magnitude of adverse events is a key issue for quality management in institutions that promote care.
\end{abstract}

Keywords: Patient safety, Adverse event, Nursing, Notification.

\section{RESUMEN}

Objetivo: Identificar los factores facilitadores y obstaculizadores que intervienen en el proceso de notificación de eventos adversos a la salud que realizan los enfermeros que laboran en un hospital de la Zona da Mata Mineira. Métodos: Estudio de caso cualitativo realizado a través de entrevista con guión semiestructurado con la participación de 25 enfermeras. Los datos se sometieron a análisis de contenido. Resultados: De los testimonios surgieron dos categorías temáticas: Aspectos facilitadores para la realización de la notificación de eventos adversos para la salud y "Notificación de eventos adversos: desafíos vividos por el equipo de

${ }^{1}$ Universidade Federal de Viçosa (UFV), Viçosa - MG. *E-mail: marilaneamaro@yahoo.com.br 
enfermería". La primera categoría destacó la comunicación efectiva, el trabajo en equipo y el aprendizaje de la notificación como aspectos que facilitan la realización de notificaciones. A su vez, la segunda categoría señaló la cultura punitiva, la falta de retroalimentación y la sobrecarga de trabajo como aspectos obstaculizadores. Conclusión: Los profesionales de la salud en su práctica diaria, experimentan la ocurrencia de eventos adversos y reconocen que el proceso de notificación es un aspecto fundamental para la promoción de la seguridad del paciente. Conocer y determinar la magnitud real de los eventos adversos es un tema clave para la gestión de la calidad en las instituciones que promueven la atención.

Palabras clave: Seguridade del paciente, Acontecimento adverso, Enfermería, Notificácion.

\section{INTRODUÇÃO}

A temática relacionada a segurança do paciente tem seu início ao longo da história com Hipócrates (460370 a.C.), que já mencionava que o cuidado poderia causar algum tipo de dano (BRASIL, 2014). Percorrendo a trajetória do cuidado, Florence Nightingale em 1852, preocupava-se com questões relacionadas ao tipo de assistência que era prestada aos soldados feridos na guerra da Criméia. O médico cirurgião Ernest Amory Codman (1869-1940), estabeleceu uma padronização que garantisse a qualidade das intervenções médicas prestadas (RAMOS ACA, 2017). Avedis Donabedian (1919), com a noção de indicadores de estrutura, processo e resultado propôs que estes seriam essenciais para a qualidade do serviço em saúde (BRASIL, 2014).

Nesse sentido, em 1990 com o relatório do Institute of Medicine: To Err is Human, a segurança do paciente ganhou maior visibilidade, uma vez que, demonstrou que a ocorrência de eventos adversos (EA) era elevada e que estes eram em sua grande maioria por causas evitáveis (KOHN LT, et al., 2000). Em 2004, a Organização Mundial da Saúde (OMS), cria a Aliança Mundial para Segurança do Paciente, com vistas a redução dos EA e definição de conceitos concernentes à temática (WHO, 2009). No ano de 2008, a Rede Brasileira de Enfermagem e Segurança do Paciente foi implementada, objetivando o cuidado seguro, a qualidade da assistência e a importância da cultura de segurança dentro das instituições de saúde (BATISTA CF, 2018). Neste contexto, em 2013, o Ministério da Saúde instituiu o Programa Nacional de Segurança do Paciente através da portaria $n^{\circ}$ 529/13 (BRASIL, 2013).

$\mathrm{Na}$ contemporaneidade a segurança do paciente ainda é uma questão preocupante uma vez que, a falha no gerenciamento dos serviços prestados pode levar a danos irreversíveis ao cliente, maior tempo de internação hospitalar, maiores gastos para a instituição e afastamento do trabalho (MOURA RS, et al., 2018). Assim, a segurança do paciente compreende a redução a um mínimo aceitável, o risco de dano desnecessário associado ao cuidado de saúde (BRASIL, 2014). Esta deve ser encarada como um conjunto de ações que visam promover e minimizar os riscos de EA e tem participação fundamental nos processos que garantam a melhoria da qualidade da assistência prestada nas organizações de saúde (WEGNER W, et al., 2016).

Nesse contexto, abrange-se também a concepção de EA, que pode ser entendido como incidente que causa dano de ordem biopsicossocial ao cliente, decorrente da assistência do cuidado prestado e não possui relação com o quadro natural da doença de base (LIMA KP, et al., 2017; SILVA AT, et al., 2016). No Brasil, dados apontam que a cada três minutos, mais de dois brasileiros vão a óbito em consequência de erros relacionados a assistência em saúde (COUTO RC, et al., 2016). Ressalta-se que vários fatores podem colaborar para a ocorrência dos EA como: materiais escassos, condições precárias de trabalho, não cumprimento de normas da organização, sobrecarga de trabalho, estresse, falta de supervisão de enfermagem, uso de inúmeras tecnologias, inexperiência profissional e falta de comunicação efetiva entre a equipe profissional (HINRICHSEN SL, et al., 2017; SILVA EKA, et al., 2018).

Um dos grandes desafios atualmente para garantir que o processo do cuidar em saúde ocorra de maneira segura, reside nas notificações de EA. A notificação fornece subsídios para o reconhecimento dos erros, permite sua monitorização, viabiliza espaços de aprendizagem na instituição de saúde para redução de tais ocorrências e fomenta a criação de uma cultura de segurança (MASCARENHAS FAS, et al., 2019).

Contudo, o cenário da realização das notificações ainda tem como principal protagonista o profissional enfermeiro uma vez que este, ocupa a linha de frente no cuidado a saúde resultando em uma única categoria profissional se responsabilizando pela segurança do paciente (FURINI ACA, et al., 2019). 
Destarte, se torna essencial desmistificar a ideia centrada no profissional enfermeiro como único responsável na execução das notificações, sendo esta uma competência de toda a equipe multiprofissional; quebrar o paradigma de caráter punitivo existente nas instituições de saúde, o que contribui para a subnotificação dos EA ocorridos e proporcionar a compreensão adequada pelos trabalhadores de que, a notificação tem por finalidade subsidiar o planejamento e gerenciamento de ações com vistas a mitigar a ocorrência de futuros erros (SIMAN AG, et al., 2017).

Neste contexto, a questão que norteou este estudo foi: quais são os fatores facilitadores e dificultadores envolvidos no processo das notificações de eventos adversos em saúde realizadas pelos enfermeiros? Assim, o objetivo desta pesquisa foi identificar os fatores facilitadores e dificultadores envolvidos no processo de notificação de eventos adversos em saúde realizadas pelos enfermeiros que atuam em um hospital da Zona da Mata Mineira.

\section{MÉTODOS}

Trata-se de um estudo de caso qualitativo, que tem por objetivo analisar uma unidade social, buscando entender os fenômenos sociais complexos e responder "como" e "por que" eles ocorrem. Esse método de pesquisa foi escolhido pois permite o pesquisador captar características significativas presentes em contexto real (YIN RK, 2015). A unidade de análise foi um hospital da Zona da Mata Mineira. Este hospital é de médio porte, considerado de natureza filantrópica sem fins lucrativos, credenciado como hospital-escola e referência de atendimento em sua microrregião.

O núcleo de segurança do paciente foi instituído no hospital em 10 de março de 2016 (BRASIL, 2013). A instituição possui 25 setores, dispõe de 106 leitos, sendo 6 leitos de ortopedia, 15 leitos de clínica masculina, 16 leitos de clínica feminina, 16 leitos de maternidade, 22 leitos de apartamentos A, 6 leitos de apartamentos B, 11 leitos de pediatria, 6 leitos de UTI adulto e 8 leitos de UTI neonatal. A população atendida pelo serviço é caracterizada de $80 \%$ SUS e $20 \%$ privado (particular e convênio).

Como critérios de inclusão dos participantes elegeram-se: ser enfermeiro coordenador ou assistencial e trabalhar a pelo menos seis meses na instituição. Foi considerado como critérios de exclusão: enfermeiros afastados do cargo por qualquer motivo no período da coleta de dados e aqueles que não ocupavam cargos de assistência ou coordenação de setor. Portanto os participantes do estudo foram 25 enfermeiros, sendo 18 enfermeiros assistenciais e 7 enfermeiros coordenadores.

A coleta de dados foi realizada no mês outubro de 2018, por meio de entrevista com roteiro semiestruturado elaborado pelos autores do estudo contendo perguntas de identificação do perfil dos participantes (nome, idade, sexo, se possui pós-graduação, tempo de formação e tempo de trabalho na instituição).

O roteiro também continha perguntas abertas como: para você o que é um EA? Qual a sua atitude diante uma situação na qual você percebe que o paciente foi exposto a algum risco? Quais informações são relatadas nas notificações? Você foi orientado quanto ao preenchimento do formulário de notificação? Qual a importância de realizar as notificações? Sua equipe realiza as notificações? Como você motiva sua equipe a realizar as notificações? Quais tipos de notificações são mais frequentes no setor no qual você trabalha? Na sua opinião, o que dificulta a realização das notificações? E o que favorece a realização das notificações? Qual feedback a equipe de enfermagem recebe a respeito das notificações realizadas? Como você acredita que as notificações podem auxiliar na prevenção de um EA? Como elas contribuem para melhorar a qualidade da assistência?

As entrevistas foram gravadas e, posteriormente, transcritas na íntegra. As entrevistas foram realizadas no próprio hospital, após o plantão ou em seus intervalos, onde os participantes foram convidados a dirigiremse a um local reservado e expuseram suas vivências com tranquilidade e segurança.

Para análise dos dados foi realizada a técnica de análise de conteúdo que propõe uma sequência para análise baseada nas seguintes etapas: pré-análise, exploração do material e tratamento dos resultados, inferência e interpretação (BARDIN L, 2011). 
Após a transcrição das entrevistas, foi realizada leitura atenta para apreender as unidades de registro significativas, visando atender os objetivos da pesquisa. Em seguida, foi feita a categorização e a interpretação dos dados permeada pela literatura relacionada à temática, de modo a permitir a discussão entre os achados do estudo e o conhecimento científico existente.

Para preservar 0 anonimato dos profissionais, os mesmos foram identificados com as letras EA (enfermeiros assistenciais), EC (enfermeiro coordenador) e atribuído numeração arábica, o qual indica a ordem em que foram realizadas as entrevistas (EA1, EA2, EA3, EC1, EC2...).

O estudo foi conduzido segundo as normas da Resolução 466/2012 do Conselho Nacional de Saúde, tendo sido aprovado pelo Comitê de Ética em Pesquisa com Seres Humanos da Universidade Federal proponente parecer $n^{\circ}: 2.416 .291$.

\section{RESULTADOS E DISCUSSÃO}

Em relação às características dos participantes do estudo, verificou-se que dentre os vinte e cinco entrevistados, dezoito eram do sexo feminino. O participante mais jovem tinha 23 anos e o mais velho 43 anos. O tempo de formação variou de um ano e sete meses a 16 anos; destes, dez possuíam pós-graduação e o tempo de atuação dentro da instituição de saúde foi de seis meses a 13 anos.

A partir da compreensão dos depoimentos emergiram-se duas categorias temáticas: "Aspectos facilitadores para a realização da notificação de eventos adversos à saúde"; "Notificação de eventos adversos: desafios vivenciados pela equipe de enfermagem".

\section{Aspectos facilitadores para a realização da notificação de eventos adversos à saúde}

Esta categoria aponta os principais fatores que facilitam a realização das notificações na instituição, tais como: a comunicação eficaz, o trabalho em equipe e o aprendizado a partir da notificação. Tais aspectos foram considerados pontos favoráveis para a realização das notificações.

"Assim eu tenho bastante liberdade com todos eles. Qualquer evento eles me comunicam. Eu nunca soube assim de coisa escondida não, eles sempre me falam, porque têm liberdade". (EA25)

"Eu acho que um facilitador é o próprio fato deles relatarem para gente. Se eles falham, eles não tem medo de falar, sabe! Assumem o que estão fazendo". (EA18)

"Porque assim, eu procuro sempre trabalhar como equipe. Eu peço que eles confiem em mim. Eu falo assim "gente se acontecer qualquer coisa me avisa" porque é importante né? Nós sabermos para a gente tomar conta e depois não ir informações erradas como se nós estivéssemos fazendo alguma coisa." (EA12)

"Eu sempre dou a liberdade para eles. Eu não me coloco na frente deles, como se eu fosse melhor que eles não! Eu coloco que aqui a gente é uma equipe e tem que trabalhar junto inclusive nos erros. Quando acontece eles tem a liberdade de chegar, de contar e aí a gente conversa e resolve como fazer com essa situação." (EA4)

"A partir do momento que você notifica, vai estar contribuindo com o levantamento de dados para através de análise saber onde está sendo o erro. Onde acontece mais o erro, quais os tipos de problemas mais comuns, quais as formas passíveis de resolver." (EA24)

\section{Notificação de eventos adversos: desafios vivenciados pela equipe de enfermagem}

Esta categoria apresenta principais desafios vivenciadas pelos enfermeiros que possibilitam a ocorrência de erros. Dentre estes destacam-se: a cultura punitiva, a falta de retorno/feedback após a realização da notificação, as falhas nas barreiras (sejam elas estruturais e/ou no processo de trabalho) e a sobrecarga de trabalho do enfermeiro. Os trechos que seguem demonstram o exposto: 
"Às vezes eu acho que eles têm medo de relatar com medo de sofrer algum tipo de punição ou algum tipo de penalidade, por causa de um evento que às vezes nos faz sentir culpados." (EA7)

"Muitas vezes tem um pouco de medo. Tem que ser um trabalho diário com isso. Porque eles ficam com medo às vezes, de fazer uma notificação e prejudicar um colega ou eles mesmo fazer a notificação e se prejudicarem. Então, assim, eles ficam bem à mercê com esse medo sabe." (EC14)

\footnotetext{
"Para te ser bem sincero o feedback não é muito positivo não. Às vezes já fiz notificações e não tive nenhum retorno, nem se foi positivo ou foi negativo, sinceramente." (EA23)

"Eu não tive feedback até hoje da notificação. O que acontece: a gente preenche e ela vai para a $\mathrm{CCIH}$, mas a gente não teve um feedback dessa notificação. Então assim, eu acho essa parte ainda é falha." (EC2)
}

"Mas já aconteceu por exemplo, uma situação em que a gente teve várias etapas dessa parte da segurança que foram quebradas. Então, uma determinada medicação veio da farmácia com uma concentração diferente do que a gente usava aqui, chegou a ser feita algumas doses porque a ampola era exatamente igual! A fonte, a cor do escrito e as letras eram muito pequenas, ninguém percebeu. O problema é que furou várias barreiras; então furou primeiro a barreira do laboratório, que fez uma medicação com uma concentração muito diferente e uma embalagem exatamente igual. Furou a farmácia, que dispensa as medicações. Então, esse medicamento veio errado da farmácia, entrou a questão da equipe de enfermagem que foi administrar e não conferiu a concentração da ampola." (EC17)

"A gente cai na mesma sobrecarga de trabalho. Já tem tanta coisa para fazer o dia inteiro que por mais que seja rápido notificar a gente não pega para fazer, porque está muito ocupado em outras coisas." (EA5)

\section{DISCUSSÃO}

Os achados do estudo demonstraram que os profissionais de saúde em sua prática cotidiana, vivenciam a ocorrência de EA e reconhecem que o processo de notificação é um aspecto fundamental para a promoção da segurança do paciente. Contudo, o cenário da realização das notificações ainda tem como principal protagonista o profissional enfermeiro uma vez que este, ocupa a linha de frente no cuidado a saúde resultando em uma única categoria profissional se responsabilizando pela segurança do paciente (FURINI ACA, et al., 2019).

Dados da pesquisa corroboram com a literatura no tocante ao trabalho em equipe e a comunicação serem elementos chave para uma cultura segura (PENA MM e MELLEIRO MM, 2018). Por meio dos depoimentos dos participantes, o trabalho em equipe foi ressaltado como aspecto positivo no que tange o registro das notificações. Este aspecto é salutar dentro das unidades prestadoras do cuidado uma vez que, proporciona a criação de uma cultura de segurança positiva bem como a promoção de práticas seguras em saúde. Não obstante, a comunicação entre membros da equipe multidisciplinar, quando realizada adequadamente, influência de modo significativo na continuidade do cuidado e na troca de informações sobre todo processo assistencial (LIMA SMS, et al., 2018).

O estabelecimento de uma comunicação aberta e sem falhas entre os gestores institucionais e os profissionais da unidade de saúde, é elemento crucial para a promoção de um cuidado seguro. Esta, proporciona uma compreensão sobre as ocorrências dos EA, viabiliza o conhecimento sobre em quais aspectos se deve atuar e garante o planejamento de estratégias de prevenção de futuros EA (BICA TFS, et al., 2017). Por meio do relato dos participantes foi possível inferir que, quando a comunicação ocorre de maneira clara e efetiva entre a equipe, influencia beneficamente nas condições as quais o cuidado é prestado, repercutindo significativamente na qualidade da assistência (GONÇALVES AF, et al., 2019). 
Dentre as barreiras no processo de notificação, os participantes do estudo apontaram a culpa, o medo de sofrer represálias e a punição como fatores condicionantes a não realização da mesma. Estes sentimentos, evidenciam o paradigma de cultura punitiva que ainda é praticado e disseminado dentro das organizações de saúde, o que fomenta a construção de uma cultura institucional de não notificação dos EA bem como, um clima de insegurança do paciente (ARAÚJO JS, et al., 2016).

A literatura evidenciou que, quando a instituição de saúde prima pela cultura organizacional baseada em nomear culpados e puni-los, desconsiderando que o erro é uma conjunção entre aspectos envolvendo processos, estrutura e sistemas dentro de uma organização, corrompe o fluxo de informações que pode ser gerado a partir das notificações, cria sistemas inseguros de saúde culminando em maior possibilidade da ocorrência de erros (MASCARENHAS FAS, 2017; SILVA ACAB e ROSA DOS, 2016).

Vale ressaltar que a ocorrência de EA nas instituições de saúde, bem como a prática de notificar, devem subsidiar espaços para a aprendizagem de toda a equipe multiprofissional, a fim de quebrar o caráter punitivo que é denotado a esta prática, bem como estabelecer uma cultura de notificação nos serviços de saúde e por conseguinte, otimizar a implantação de estratégias por parte dos gestores institucionais com vistas a minimizar a possibilidade futura destes EA ocorrerem (ALVES MFT, et al., 2019).

A adoção de uma cultura organizacional segura requer a corresponsabilização entre gestores institucionais e profissionais (SARTOR GD, et al., 2016). Quando ocorre esse compartilhamento de responsabilidades, um ambiente de aprendizagem é favorecido, oportunizando espaços para que os profissionais exponham suas falhas, compartilhem lições com a equipe multiprofissional, encorajando-os a identificar condições de insegurança a clientela (MOTA GCHF, 2018).

Outro dado relevante apontado neste estudo vai ao encontro da literatura, no que tange a afirmação de que, os aspectos estruturais dentro de uma organização de saúde colaboram para práticas inseguras do cuidado, proporcionando um ambiente de práticas assistenciais inseguras. Dentre estes fatores vale ressaltar, a sobrecarga de trabalho vivenciada pelos profissionais no exercício de suas atribuições. Esta, favorece sobremaneira a probabilidade de que EA ocorram e consequentemente, reverbera ao não relato por parte do profissional bem como o não registro dos mesmos (COSTA DB, et al., 2018; REIS GAX, et al., 2017; VIMIEIRO VL, 2017).

De acordo com os depoimentos, a falta de retorno/feedback por parte dos gestores institucionais, foi elencado como um aspecto dificultador da realização das notificações. Se a instituição tem por finalidade apenas arquivar os dados oriundos das notificações, sem sua análise, discussão com os profissionais e a implementação de estratégias para a melhoria da qualidade do cuidado prestado (LEMOS GC, et al., 2018), os profissionais enxergam que não houve reconhecimento do tempo que foi dispendido para o registro, não se sentem como colaboradores dentro da organização de saúde e a desmotivação em continuar relatando as ocorrências se torna mais crescente (HOFFMAN P , 2016).

É imperativo que a equipe envolvida na gestão promova a realização de feedback/retorno, a disponibilização de informações decorrentes das análises dos EA, bem como das medidas a serem implementadas e o impacto gerado sob a assistência prestada. Este retorno possibilita o reconhecimento pelos profissionais como agentes colaboradores para uma assistência segura e de qualidade assim como, promotores da cultura de segurança dentro da instituição de saúde (MASCARENHAS FAS, 2017; HOFFMAN P, 2016; REIS GAX, et al., 2017).

$O$ registro das notificações permite o conhecimento da real magnitude dos EA, o quanto estes impactam na vida dos sujeitos submetidos aos cuidados em saúde, possibilita o compartilhamento da responsabilidade entre gestores e profissionais de saúde no tocante a traçar metas que minimizem futuras ocorrências (HOFFMAN P, 2016). Vale ressaltar que, a notificação de EA é de responsabilidade de todos os profissionais que ocupam cotidianamente a linha de frente do cuidado como, enfermeiros, médicos, técnicos e auxiliares de enfermagem entre outros (FIGUEIREDO ML e D'INNOCENZO M, 2017).

A adoção e implementação de práticas educacionais de forma contínua nas organizações de saúde se fazem necessárias para estimular o registro das notificações bem como, viabilizam melhorias nas práticas em 
saúde. Tais práticas podem ser efetuadas por meio da realização de ações de educação permanente e educação continuada de toda a equipe multiprofissional. Estas estratégias fomentam dentro dos sistemas promotores do cuidado, meios para a percepção dos profissionais da importância do ato de notificar e corrobora na disseminação de uma cultura segura para o paciente (STELLUTE G, et al., 2018; MOREIRA I, 2018).

\section{CONCLUSÃO}

Neste estudo foi possível compreender as facilidades e dificuldades que permeiam o registro das notificações dos EA. Conhecer e determinar a real magnitude dos EA, é questão primordial para uma gestão de qualidade nas instituições promotoras do cuidado. Nesse sentido, o desafio dos gestores institucionais no monitoramento dos EA, reside em transformar o paradigma punitivo em um novo modelo voltado a trabalhar o erro de modo coletivo, compactuando da ideia de que este é multifatorial, garantindo a aprendizagem de toda a equipe multiprofissional por meio de estratégias de capacitação contínua com vistas a implementar a segurança do paciente.

\section{REFERÊNCIAS}

1. ALVES MFT, et al. Motivos para a não notificação de incidentes de segurança do paciente por profissionais de saúde: revisão integrativa. Ciênc. Saúde Coletiva, 2019; 24(8): 2895-2908.

2. ARAÚJO JS, et al. Conhecimento dos enfermeiros sobre eventos adversos e os desafios para a sua notificação. Cogitare enferm, 2016; 21(4): 01-08.

3. BARDIN L. Análise de conteúdo/Laurence Bardin: tradução Luiz Antero Reto, Augusto Pinheiro. 7ª ed. São Paulo: 2011.

4. BATISTA CF. Fatores que influenciam a ocorrência de eventos adversos na unidade de terapia intensiva. Rev Eletr Evid e Enferm, 2018; 2(1): 15-37.

5. BICA TFS, et al. Características dos incidentes de segurança do paciente notificados em uma unidade de terapia intensiva pediátrica. Rev enferm UFPE on line, 2017, 11(suplemento 10): 4206-16.

6. BRASIL. Ministério da Saúde. Documento de Referência para o Programa Nacional de Segurança do Paciente/Ministério da Saúde; Fundação Oswaldo Cruz; Agência Nacional de Vigilância Sanitária, Brasília, 2014.

7. BRASIL. Ministério da saúde. Portaria nํ529, de 1ำ de abril de 2013. Institui o Programa Nacional de Segurança do Paciente (PNSP). Diário Oficial da União, Brasília, 2013.

8. BRASIL. Ministério da Saúde. Resolução da Diretoria Colegiada no 36, de 25 de julho de 2013. Institui ações para a segurança do paciente em serviços de saúde e dá outras providências. Diário da união, Brasília, 2013.

9. COSTA DB, et al. Cultura de segurança do paciente: avaliação pelos profissionais de enfermagem. Texto Contexto Enferm, 2018; 27(3): e2670016.

10. COUTO RC, et al. Instituto de Estudos de Saúde Suplementar - IESS. Erros acontecem: A força da transparência no enfrentamento dos eventos adversos assistenciais em pacientes hospitalizados. Belo Horizonte, 2016.

11. FIGUEIREDO ML, D'INNOCENZO M. Eventos adversos relacionados às práticas assistenciais: uma revisão integrativa. Revista eletrônica trimestral de enfermeria, 2017; 47:605-620.

12. FURINI ACA, et al. Notificação de eventos adversos: caracterização dos eventos ocorridos em um complexo hospitalar. Rev Gaúcha Enferm. 2019; 40(esp): e20180317.

13. GONÇALVES AF, et al. Estratégias e implicações da segurança do paciente na prática do cuidado de enfermagem. Braz J Hea Rev, 2019; 2(1): 378-393.

14. HINRICHSEN SL, et al. Percepção da equipe de enfermagem sobre fatores causais de quase-falhas (near miss) no atraso de medicamentos através da análise do tipo de efeito e falha (Failure Mode and Effects Analysis) - FMEA. Revista administração em saúde, 2017; 17(66): 1-18.

15. HOFFMAN P. Sistema de notificação de eventos adversos e/ou incidentes em saúde: software-protótipo. Dissertação (mestrado Profissional) - Universidade Federal de Santa Catarina, Florianópolis, 2016.

16. KOHN LT, et al. To err is human: building a safer health system. Committee on quality of health care in America, Institute of Medicine. Washington (DC): National Academy Press, 2000.

17. LIMA KP, et al. Fatores contribuintes para ocorrência de eventos adversos em unidade de terapia intensiva: perspectiva do enfermeiro. Rev enferm UFPE on line, 2017; 11(3): 1234-43.

18. LIMA SMS, et al. Percepção dos profissionais de saúde das limitações à notificação do erro/evento adverso. Revista Enfermagem Referência. Série IV (19): 99-106.

19. LEMOS GC, et al. A cultura de segurança do paciente no âmbito da enfermagem: reflexão teórica. Revista de enfermagem Centro-Oeste Mineiro, 2018; 8: e2600.

20. MASCARENHAS FAS, et al. Facilidades e dificuldades dos profissionais de saúde frente ao processo de notificação de eventos adversos. Texto e Contexto Enfermagem, 2019; 28: e20180040.

21. MASCARENHAS FAS. Notificação de eventos adversos em saúde e queixas técnicas: construção de infográfico animado educacional. Dissertação (mestrado profissional) - Universidade Federal de Santa Catarina, centro de ciências da saúde, programa de pós-graduação em gestão do cuidado em enfermagem, Florianópolis, 2017. 
22. MOREIRA I. Notificação de eventos adversos: o saber e o fazer de enfermeiros. Dissertação (mestrado) - Universidade Federal de Goiás, Instituto de Patologia Tropical e Saúde Pública, Programa de Pós-graduação em Saúde Coletiva (profissional), Goiânia, 2018.

23. MOTA GCHF. A percepção de profissionais de saúde sobre cultura de segurança do paciente em hospital universitário. Dissertação (para título de mestre) - Faculdade de Ciências Farmacêuticas, Universidade de São Paulo, 2018.

24. MOURA RS, et al. Eventos Adversos ocasionados pela assistência de enfermagem: noticiados pela mídia. Rev enferm UFPE on line, 2018; 12(6): 1514-23.

25. PENA MM, MELLEIRO MM. Eventos adversos decorrentes de falhas de comunicação: reflexões sobre um modelo para transição do cuidado. Rev Enfem UFSM, 2018: 8(3): 616-625.

26. RAMOS ACA. Educação Permanente e a Segurança do Paciente: uma Revisão Integrativa da Literatura. Monografia (especialização) - Instituto Oswaldo Cruz, Pós-graduação em Ensino em Biociências e Saúde, Rio de Janeiro, 2017; $83 \mathrm{p}$.

27. REIS GAX, et al. Implantação das estratégias de segurança do paciente: percepções de enfermeiros gestores. Texto Contexto Enferm, 2017; 26(2): e00340016.

28. SARTOR GD, et al. Segurança do paciente em hospitais de grande porte: panorama e desafios. Cogitare Enferm. Santa Catarina, 2016; 21(5): 01-08.

29. SILVA ACAB, ROSA DOS. Cultura de segurança do paciente em organização hospitalar. Cogitare Enfermagem, 2016; 21(5): 01-10.

30. SILVA AT, et al. Assistência de enfermagem e o enfoque da segurança do paciente no cenário brasileiro. Saúde debate, 2016; 40(111): 292-301.

31. SILVA EKA, et al. Eventos adversos (EA): conhecimento dos profissionais de enfermagem de um município do interior da Amazônia brasileira. Revista Científica da Faculdade de Educação e Meio Ambiente- FAEMA, 2018; 9(2): $686-691$.

32. SIMAN AG, et al. A prática de notificação de eventos adversos em um hospital de ensino. Rev Bras Enferm. Brasília, 2017; 70(1): 146-154.

33. STELLUTE G, et al. Conhecimento de enfermeiros de unidades de terapia intensiva acerca da notificação de eventos adversos. Arq Med Hosp Fac Cienc Med Santa Casa São Paulo, 2018; 63 (2):77-84.

34. VIMIEIRO VL. Fatores determinantes da subnotificação dos eventos adversos pela equipe de enfermagem. Trabalho de conclusão de curso de pós-graduação - Faculdade Método de São Paulo, Belo Horizon te, 2017.

35. WEGNER W, et al. Educação para cultura da segurança do paciente: Implicações para a formação profissional. Esc Anna Nery, 2016; 20(3): e20160068.

36. WHO. WORLD HEALTH ORGANIZATION. Global priorities for patiente safety research, 2009.

37. YIN RK. Estudo de caso: Planejamento e métodos. 5a ed. Porto Alegre: Bookman, 2015. 